\title{
Efficacy and Safety of Amphotericin B with Autologous Serum for Fungal Corneal Ulcer
}

\author{
Yongjing Ji1, Yongqiang Ji2 and Fang Zhang3
}

\begin{abstract}
Objective: To observe the clinical efficacy and safety of injection therapy of amphotericin B combined with autologous serum in the treatment of fungal corneal ulcer.

Study Design: An experimental study.

Place and Duration of Study: Department of Ophthalmology, Jinan Second People's Hospital, Shandong Province, China, from July 2013 to June 2017.

Methodology: Patients diagnosed with monocular fungal corneal ulcer were divided randomly into observation group and control group, 48 cases in each group. Control group was treated with amphotericin B alone. Observation group was treated with autologous serum injection on the basis of the control group treatment. The improvement time of clinical symptoms such as hypopyon, corneal ulcer, foreign body sensation and photophobia were observed in two groups. The clinical efficacy and adverse reactions were compared between the two groups.

Results: The total effective rate of the observation group was higher than that of the control group $(p=0.011)$. After treatment, the improvement time of the symptoms such as hypopyon, corneal ulcer, foreign body sensation, and photophobia was shorter in the observation group than the control group (all $p<0.001)$. There was no significant difference in adverse reaction rate between the two groups $(p=0.557)$.

Conclusion: Injection therapy of amphotericin B combined with autologous serum has significant efficacy for fungal corneal ulcer, which can effectively improve the clinical symptoms with fewer adverse reactions and safe and reliable results, thus worthy of promotion.
\end{abstract}

Key Words: Amphotericin B, Serum, Injection, Corneal ulcer, Clinical efficacy.

\section{INTRODUCTION}

Fungal corneal ulcer is one of the most common infective corneal ulcers. The incidence of this disease is high, and the course of disease is relatively long. Lack of sensitive and effective antifungal drugs in clinical treatment is the main reason for the treatment failure of this disease.1,2 Recently, the prevalence of fungal corneal ulcers has been increasing year by year. ${ }^{3}$ Once medical treatment of patients with fungal corneal ulcers is not effective, thus surgery is required. 4 Therefore, timely and effective medical treatment in the early stage of infection of patients with fungal corneal ulcer is of great importance. Most antifungal agents, currently, have more side effects and limited application; fungi of different species have different sensitivities to different antifungal agents. 5

Amphotericin $B$ is a broad-spectrum antifungal drug that inhibits cryptococcus neoformans, candida albicans, and

1 Department of Ophthalmology, Jinan Second People's Hospital, Shandong Province, 250000, China

2 Department of Medicine $/{ }^{3}$ Medical Record, Jinan Bus Hospital, Shandong Province, 250000, China

Correspondence: Fang Zhang, Department of Medical

Record, Jinan Bus Hospital, Shandong Province, 250000,

China

E-mail:lf39r57808@163.com

Received: February 08, 2018; Accepted: September 26, 2018 microsporum sphaeroides. ${ }^{6,7}$ Autologous serum adjuvant treatment of fungal corneal ulcer can alleviate corneal irritation, reduce inflammatory reactions, and promote corneal ulcer healing, which can promote lesion recovery and tissue healing. 8,9

This study was carried out to investigate the application value of amphotericin B combined with autologous serum injection in the treatment of fungal corneal ulcer, in order to provide a reference for the clinical treatment of fungal corneal ulcer.

\section{METHODOLOGY}

This study was conducted at the Department of Ophthalmology, Jinan Second People's Hospital, Shandong Province, China, from July 2013 to June 2017. A total of 96 patients diagnosed with fungal corneal ulcer were selected as subjects. Inclusion criteria were early foreign body sensation, eye pain, photophobia, lacrimation, and other irritating symptoms; corneal lesions were offwhite, slightly elevated, rough surfaced and easy to scrape; with possible iridocyclitis, offwhite sticky hypopyon; no response use of antibiotic treatment; and hyphae in corneal scraping staining. Exclusion criteria were severe heart, liver, kidney, lung and other organ functional disease or malignant tumor; drug allergy, poor compliance; pregnant or lactating women; those with mental disorders and unable to communicate normally. The study was approved by the 
Hospital Ethics Committee, and all patients participated in the study voluntarily.

The patients were divided into observation group and control group according to random number table method, 48 cases in each group. The two groups underwent surgical debridement. After conventional anesthesia and disinfection, hyphae and necrotic tissues on the ulcer surface were scraped. Adjuvant therapy of routine corneal nutrition drugs was provided after operation. The control group was treated with amphotericin $B$. The amphotericin B was prepared as eye drops at the concentration of $0.25 \% \sim 0.3 \%$ for $1 \sim 2$ drops/ time for 1 hour, with 30 days' course of treatment.

The observation group was treated with autologous serum injection in addition to the control group treatment. Twelve $\mathrm{mL}$ of autologous venous blood was drawn from the patients, placed in a sterile serum tube for 30 minutes, centrifuged at $4000 \mathrm{r} / \mathrm{min}$ after blood coagulation, and the yellow serum was extracted by syringe and placed in sterile eye drop bottles for four days under low temperature preservation. Serum collection date was marked to prevent expiration. Subconjunctival injection of autologous serum was performed, each $0.6 \mathrm{~mL}, 1$ time / 2 days, with treatment course of 30 days.

Improvement time of clinical symptoms such as hypopyon, corneal ulcers, foreign body sensation, photophobia were observed and recorded during the course of treatment. During the treatment, blood and urine routine, liver and kidney function detection were routinely conducted once a month, and the adverse reactions of the two groups were observed and recorded. Cured was considered corneal ulcers and hypopyon disappeared completely, clinical symptoms improved significantly, fluorescein staining was negative and pathogenic fungi were not found in ulcer parts in laboratory tests. It was considered markedly effective when most corneal ulcer disappeared, hypopyon reduced by $>50 \%$, clinical symptoms improved, fluorescein staining was negative, and pathogenic fungi were not found in ulcer parts in laboratory tests. It was considered invalid when ulcer and hypopyon either disappeared in small area or did not disappear, fluorescein staining was positive, and pathogenic fungi were still found in ulcer parts in laboratory tests. Total efficiency was defined as the sum of the cure rate and markedly effective rate.

All data were analysed by SPSS 21.0 software package. The normalised measurement data were expressed as " $\bar{x} \pm s "$, and the independent sample t-test was used to compare the two groups. The count data was expressed in terms of frequancies with percentages, and the two groups were compared by $\chi^{2}$ test with $p<0.05$ indicating statistically significant difference.

\section{RESULTS}

All 96 patients had monocular disease, including 54 $(56.25 \%)$ males and $42(43.75 \%)$ females, with an average age of $42.57 \pm 2.41$ years; average course of disease $48.35 \pm 3.16$ days. The left eye was affected in 47 cases and right eye in 49 cases. There were 52 $(54.17 \%)$ cases with plant trauma and 15 (15.63\%) cases with corneal foreign body removal prior to symptoms.

The total effective rate of the control group was $75.00 \%$ (36 cases); that of the observation group was $93.75 \%$ (45 cases). The total effective rate of the observation group was higher than that of the control group, with statistically significant difference $(p=0.011$, Table I).

After treatment, the clinical symptoms and signs improved to some extent in both the groups. The improvement time of the relevant symptoms such as hypopyon, corneal ulcer, foreign body sensation, photophobia was shorter in the observation group than the control group, with statistically significant difference (all $p<0.001$, Table II).

Neither group of patients exited the study because of the treatment regime intolerance or had functional impairment of organs such as liver and kidney. In the observation group, gastrointestinal reaction was $2.08 \%$ ( 1 case), which was not treated. The symptom disappeared gradually after the treatment was stopped. In the control group, gastrointestinal reaction was $4.17 \%$ ( 2 cases). Comparison of adverse reaction rates between the two groups were not statistically significant $(p=0.557)$.

Table I: Comparison of clinical outcomes between two groups after treatment.

\begin{tabular}{lccccc}
\hline Groups & Case number & Cured & Markedly effective & Invalid & Total effective \\
\hline Control group & 48 & $17(35.42)$ & $19(39.58)$ & $12(25.00)$ & $36(75.00)$ \\
Observation group & 48 & $28(58.33)$ & $17(35.42)$ & $3(6.25)$ & $45(93.75)$ \\
p-value & & & & & 0.011 \\
\hline
\end{tabular}

Table II: Comparison of clinical symptom improvement time of the two groups.

\begin{tabular}{l|c|cccc}
\hline Groups & Case number & \multicolumn{4}{|c}{ Clinical symptom improvement time } \\
\cline { 2 - 5 } & & Hypopyon (d) & Corneal ulcers (d) & Foreign body sensation (d) & Photophobia (d) \\
\hline Control group & 48 & $21.34 \pm 3.69$ & $22.83 \pm 3.85$ & $17.64 \pm 3.53$ & $24.61 \pm 4.61$ \\
Observation group & 48 & $13.45 \pm 1.85$ & $15.53 \pm 1.38$ & $11.09 \pm 1.91$ & $17.79 \pm 5.98$ \\
p-value & & $<0.001$ & $<0.001$ & $<0.001$ & $<0.001$ \\
\hline
\end{tabular}




\section{DISCUSSION}

Keratoplasty is currently the most effective means of clinical treatment of fungal corneal ulcer. However, compared to keratoplasty, drug therapy has a lower physical and economic burden on the patient and does not present a risk of immune rejection after keratoplasty. ${ }^{10,11}$ Therefore, it is a feasible treatment to pay attention to the effective drug treatment in the early stage of fungal corneal ulcer, and it can greatly avoid the physical pain and economic burden on the patient. In recent years, the rational selection of antifungal drugs and improving the effectiveness of antifungal drugs have received increasing attention.12,13 Among them, amphotericin $B$ is a polyene antifungal drug and is a firstline antifungal drug for clinical use. ${ }^{14}$ Related data show that amphotericin B is suitable for corneal infection caused by a variety of fungi. Its mechanism of action is that it can bind to sterols on the cytoplasmic membrane of sensitive fungi, then micropores form on the membrane, and the permeability of the membrane changes, so that the electrolyte and amino acids in the cells are extravasated, thereby destroying the normal metabolism of the fungus and inhibiting its growth. ${ }^{15,16}$ The autologous serum is obtained by centrifuging the patient's own venous blood. It is freshly prepared and contains no preservatives. ${ }^{17}$ Autologous serum contains a large amount of albumin, globulin, antibodies and various growth factors. Macroglobulin in plasma is an effective collagenase inhibitor that prevents corneal ulceration and perforation. The serum antibody content in patients with keratitis is significantly higher than that in normal people. There are a large number of antibodies, complements and interferons, which can improve the body's immunity and enhance the ability of corneal tissue to resist the attack of fungitoxicity. ${ }^{18}$ The serum also contains ammonia, carboxyls, peptides, nucleic acids, sugars and organics, which can improve tissue nutrition, stimulate cell regeneration and accelerate tissue repair. ${ }^{19}$ In addition, various growth factors in serum can promote the healing of the corneal epithelial layer and the stromal layer. ${ }^{20}$

In this study, it is found that the total effective rate of the observation group was higher than that of the control group. It is indicated that amphotericin B combined with autologous serum injection has better efficacy. It is suggested that the combination of amphotericin B and autologous serum has a synergistic effect, and the antifungal efficacy of the combination of the two drugs is stronger than that of amphotericin B alone. At the same time, in the study, it is found that the clinical symptoms and signs of the two groups of patients showed improvement with different degree after treatment. The improvement time of anterior chamber abscess, corneal ulcer, foreign body sensation, photophobia and lacrimation in the observation group was significantly shortened. It indicated that amphotericin B combined with autologous serum injection can relieve the clinical symptoms of patients with fungal corneal ulcer in a short time, improve the quality of life, and accelerate the regeneration of damaged tissue. It is also found that the two groups of patients were free of liver, kidney, heart, lung and other organ damage. There was no significant difference in the adverse reaction rate between the observation group and the control group. It is further indicated that amphotericin B combined with autoantibody injection therapy has higher safety.

The limitation of this study was that the number of samples included in the study was small; and the clinical observation time was short. The patients who were treated with amphotericin B combined with autoantibody injection were not followed up for a long term to observe the occurrence of recurrence. In the future, the number of samples can be expanded and the clinical observation time can be extended for further study to clarify the efficacy of amphotericin B combined with autoantibody injection therapy.

\section{CONCLUSION}

Amphotericin B combined with autologous serum injection in the treatment of patients with fungal corneal ulcer can effectively relieve the clinical symptoms of patients in a short period of time, the effect is exact, and it has less adverse reactions which is safe and reliable, so it is worth popularising.

\section{REFERENCES}

1. Ray KJ, Lalitha P, Prajna NV, Rajaraman R, Krishnan T, Srinivasan $M$, et al. The utility of repeat culture in fungal corneal ulcer management: a secondary analysis of the multirandomized clinical trial. Am J Ophthalmol 2017; 178:157-62.

2. Chander J, Chakrabarti A, Sharma A, Saini JS, Panigarhi D. Evaluation of calcofluor staining in the diagnosis of fungal corneal ulcer: der stellenwert der calcofluor-farbung in der diagnose des pilzbedingten hornhaut-ulkus. Mycoses 1993; 36:243-5.

3. Chen Y, Yang W, Gao M, Belin MW, Hai Y, Jing Y. Experimental study on cryotherapy for fungal corneal ulcer. BMC Ophthalmol 2015; 15:29.

4. Zhang MC, Liu X, Jin Y, Jiang DL, Wei XS, Xie HT. Lamellar keratoplasty treatment of fungal corneal ulcers with acellular porcine corneal stroma. Am J Transplant 2015; 15:1068-75.

5. Matsumoto $Y$, Dogru M, Goto E, Fujishima H, Tsubota K. Successful topical application of a new antifungal agent, micafungin, in the treatment of refractory fungal corneal ulcers: report of three cases and literature review. Cornea 2005; 24:748-53.

6. Sangalli-Leite F, Scorzoni L, Alves de Paula E Silva AC, da Silva JF, de Oliveira HC, de Lacorte Singulani J, et al. Synergistic effect of pedalitin and amphotericin B against cryptococcus neoformans by in vitro and in vivo evaluation. Int $J$ Antimicrob Agents 2016; 48:504-11.

7. Khan AA, Jabeen M, Alanazi A, Khan AA. Antifungal efficacy of amphotericin $B$ encapsulated fibrin microsphere for treating 
Cryptococcus neoformans, infection in Swiss albino mice. Braz J Infect Dis 2016; 20:342-8.

8. Akyol-Salman I. Effects of autologous serum eye drops on corneal wound healing after superficial keratectomy in rabbits. Cornea 2006; 25:1178-81.

9. Janyamethakul $T$, Moleechat $P$, Gohain R, Somgird C, Pongsopavijit P, Wititkornkul B. Efficacy of autologous serum as an adjunct treatment for a melting corneal ulcer in a captive asian elephant. Thai Vet Med 2015; 45:295-9.

10. Guo H, Zhu L.The treatment of earlier recurrent fungal corneal ulcer after corneal transplantation. Chin J Practic Ophthalmol 2003; 21:117-9.

11. Idiculla $T$, Zachariah $G$, Keshav BR, Basu S. A retrospective study of fungal corneal ulcers in the south sharqiyah region in oman. Sultan Qaboos Univ Med J 2009; 9:59-62.

12. Ray A. Efficacy of topical and systemic itraconazole as a broad-spectrum antifungal agent in mycotic corneal ulcer: A preliminary study. Indian J Ophthalmol 2002; 49:71-72.

13. Xie H, Peng L, Li Z, Rui L, Sun H, Wu D. Continuous intrathecal administration of liposomal amphotericin $B$ for treatment of refractory cryptococcus neoformans encephalitis: A case report. Exp Ther Med 2017; 14:780-4.

14. Adlermoore JP, Gangneux JP, Pappas PG. Comparison between liposomal formulations of amphotericin B. Med Mycol 2016; 54:223-31.

15. Hirose $\mathrm{H}$, Terasaki $\mathrm{H}$, Awaya $\mathrm{S}$, Yasuma T. Treatment of fungal corneal ulcers with amphotericin B ointment. Am J Ophthalmol 1997; 124:836-8.

16. Garcia-Valenzuela E, Song CD. Intracorneal injection of amphothericin B for recurrent fungal keratitis and endophthalmitis. Arch Ophthalmol 2005; 123:1721-3.

17. Matsumoto $Y$, Dogru M, Goto E, Ohashi $Y$, Kojima T, Ishida R, et al. Autologous serum application in the treatment of neurotrophic keratopathy. Ophthalmology 2004; 111:1115-20.

18. Ortiz AJF, Sandra PRB, Luis F. Comparison of autologous serum with a commercial product as an adjunct in the treatment of uncomplicated corneal ulceration in dogs. Revista Colombiana De Ciencias Pecuarias 2012; 25:90-6.

19. Sanz-Marco E, Lopez-Prats MJ, Garcia-Delpech S, Udaondo P, Diaz-Llopis M. Fulminant bilateral haemophilus influenzae keratitis in a patient with hypovitaminosis A treated with contaminated autologous serum. Clin Ophthalmol 2011; 5:71-3.

20. Etxebarria J, Sanz-Lázaro S, Hernáez-Moya R, Freire V, Durán JA, Morales MC, et al. Serum from plasma rich in growth factors regenerates rabbit corneas by promoting cell proliferation, migration, differentiation, adhesion and limbal stemness. Acta Ophthalmol 2017; 95:e693-705. 